\title{
COUPLED FLUIDITY/Y3D TECHNOLOGY AND SIMULATION TOOLS FOR NUMERICAL BREAKWATER MODELLING
}

\author{
Jiansheng Xiang ${ }^{1}$, John-Paul Latham ${ }^{1}$, Axelle Vire ${ }^{1}$, Elena Anastasaki ${ }^{1}$, Christopher C. Pain ${ }^{1}$ \\ FEMDEM modelling which combines the multi-body particle interaction and motion modelling (i.e. Discrete \\ Element Model, DEM) with the ability to model internal deformation of arbitrary shape (Finite Element Model, \\ FEM) has been applied to breakwater models. There are two versions of a FEMDEM solver developed; Y3D D is \\ for deformable materials and is required for dynamic and static stress analysis and Y3D-R is the rigid version often \\ used to numerically construct the armour unit packs. This paper also reports the placement protocols: POSITIT.
}

FEMDEM modelling deals with solids interactions and is one modelling component that is to be coupled to other modelling technologies e.g. CFD, interface tracking, wave models, porous media etc. so that the key fluid-solid interactions can be modelled in a full scale virtual breakwater alongside work on scaled hydraulic laboratory models and prototype structures. The latest developments of two-way coupled interactions of waves with coastal structures are also described in this paper.

Keywords: rubble-mound breakwater; Fluid-structure interaction; combined finite-discrete element method

\section{BACKGROUND}

The design of rubble mound structures is heavily based on hydraulic physical models which guide hydraulic performance and hydraulic stability of armour layers. However, due to high costs and the dependency on weather conditions full-scale measurements are impossible to achieve. The theoretical understanding of fluid-solid interaction and internal load - response within armour units is largely lacking. Numerical modelling methods are becoming increasingly important in design of rubble mound structures.

In order to carry out numerical tests, the first task is to build a virtual breakwater in a computer environment with geometries representative of real armour unit structures. However, due to accuracy limitations, the surveyed data can't be used by the FEMDEM solver. It is also a real challenge to place each unit in the volume domain of the computer model such that the initial conditions of the armour layer simulation start from an equilibrium configuration that is relevant to patterns and placement densities achieved in prototype breakwaters i.e. where units positioned in the numerical model are just touching and not overlapping (Dentale, 2009). This is because units positioned in the numerical model must not overlap or else the mechanical FEMDEM model will be unstable when the simulation begins. In previous work (Xiang et al, 2011. Latham et al 2011), an 8 Row Armour Layer System of 20 Units was manually created in an interactive placement visualisation software environment ("Mover", developed by Baird Associates). A more powerful and fully automated placement protocol, POSITIT, was developed recently. POSITIT is a generic code that has many features of a pre-processor but combines this with some of the fast mechanics of the DEM code. POSITIT has various functions, e.g. user-defined particle/unit centroid, user-defined or random particle/unit orientation, user-defined initial velocity applied to all particles/units to deposit particles/units faster, etc. The details of POSITIT are described in the section on Simulation Tools. A rigid FEMDEM version namedY3D-R in which solid multiple bodies are assumed to behave as rigid bodies was developed to be coupled with POSITIT. Several post-processing tools have been developed recently, e.g. factor of safety, coordination number, and stereographic plot etc. These tools will be described in another paper.

Coastal engineers also seek both physical and numerical models to investigate the efficiency of different geometries of rubble mound breakwaters and armour unit stability during wave energy dissipation. One major advantage of numerical studies is therefore the ability to visualise the complete internal stress distribution within the solid. Moreover, it often takes a long period of time to set-up an experimental model, which also can be expensive, whereas numerical models can be quickly set-up and are carried out on computers, or supercomputers if necessary. Furthermore, the issue of scale effects and model effects, almost impossible to resolve in scaled flume and tank experiments are less severe or absent in numerical simulation. However, the grand challenge is to model such complex system that includes the interaction of energetic storm waves breaking on complex solid structures and devices and the interaction between solid geometries.

Numerical modelling of purely fluid systems is a relatively mature research field and modelling of purely dry particulates is about 25 years old and a rapidly developing field. By contrast, the modelling of the solid's influence on the fluid motion and vice versa is very new and presents great challenges. It becomes obvious that capturing the fluid-solid interactions (FSI) accurately in numerical simulations is crucial and will enable engineers to obtain more reliable simulation results. One promising numerical

\footnotetext{
${ }^{1}$ Earth Science and Engineering Department, Imperial College London
} 
method for turbulent fluids, direct numerical simulation (DNS) has grown up quickly due to the recent dramatic growth in computing power. $\mathrm{Hu}(\mathrm{Hu}, 1996)$ applied a generalized Galerkin finite element method based on moving unstructured grids and an arbitrary Lagrangian-Eulerian (ALE) technique to simulate the motion of particles in a flowing liquid. Good stability was achieved under the assumption of no particle collisions, although the method is less suited to dense particle phases. (Glowinski et al., 2001) developed a 'fictitious domain' method in which the whole is discretized into a fixed mesh, and a superimposed solid mesh locates the particles which then respond by rigid motion. Particle contact detection and particle-particle interactions were simulated by the Discrete Element Method (DEM) invented by Peter Cundall in 1979 (Cundall and Strack, 1979). A short-range repulsive force was used to avoid overlapping of regions occupied by the rigid body. A possible drawback of these approaches is the computational expense of their fixed mesh implementation. This has encouraged several researchers to replace full Navier-Stokes flow with a Lattice-Boltzmann approximation when coupling with DEM methods (e.g.: Cook et al., 2004; Han et al., 2007). However, the regular structured mesh constrains the applications of Lattice-Boltzmann methods in complex geometry systems. Several groups combined Smoothed Particle Hydrodynamics (SPH) with DEM (e.g. Potapov et al., 2001). However, the classical DEM is limited in simulating angular/arbitrary shape of particles. This drawback constrains applications of these approaches, e.g. it is very difficult to simulate non-convex shapes of armour units using DEM codes.

In this paper we present an immersed method in order to model FSI problems. Our approach is to project relevant quantities between the two meshes, one for each phase, via a supermesh (see Farrell et al., 2011). In the proposed approach the two codes, ' $Y 3 D$ ' based on FEMDEM and the multi-purpose CFD code, 'Fluidity', are coupled to model fluid-structure interactions. Y3D captures nonlinear material properties using a finite strain formulation and also has multi-body and granular media capability. Fluidity is a multi-phase CFD code based on arbitrary unstructured finite element meshes with the capability of anisotropic dynamic mesh optimisation. A core concern with modelling relatively large moving particles (i.e. not sub-grid sized particles) is the computational expense of fluid re-meshing. Our approach is based on solving Navier-Stokes equations using a Petrov-Galerkin Finite Element method with an adaptive unstructured mesh (Pain et al., 2001), together with recently enhanced interface tracking and mesh movement algorithms (Mindel et al., 2007). This meshing technology allows the fluid to refine around the complex (possibly moving) geometries, e.g. a CORE$\mathrm{LOC}^{\mathrm{TM}}$, or seek-out the complex topology of void space in porous granular media, starting with an arbitrary coarse mesh. This step also reduces the effort of meshing around solid bodies within a fluid and thus drastically decreases the time taken to set up fluid-structure models.

In this paper, we briefly describe mathematical models including FEMDEM and Fluidity in section - "MATHEMATICAL MODELS". In section of "RESULTS", two-way coupling is illustrated through simulation of a particle accelerating to terminal velocity as it falls through a viscous liquid. One application of fluids-solids coupling related to coastal structures is illustrated. We deposit solids resembling CORE-LOC ${ }^{\mathrm{TM}}$ units on an inclined plane within the fluid computational domain (which resembles a wave-tank) and allow a wave generated by a collapsing water column to interact with them. In section of "SIMULATION TOOLS", POSITIT and Y3D-R are briefly introduced with an application of generating a realistic armour unit layer with 242 CORE-LOC $^{\mathrm{TM}}$ units. We finally draw conclusions.

\section{MATHEMATICAL MODELS}

The FEMDEM method was pioneered by Munjiza whose first working 2D FEMDEM code was developed in 1990 (Munjiza 2004). FEMDEM has proven its efficiency and reliability as a computational tool to solve problems involving transient dynamics of systems in which deformation and fracturing play an important role. In 2009, an efficient 10-noded quadratic element was developed in a format suitable for the FEMDEM by Xiang (Xiang et al. 2009). The 3D FEMDEM code (Y3D) which was developed by Xiang and Munjiza in 2009 has great potential to be applied in the field of coastal structures. In this paper FEMDEM is introduced briefly while details can be found elsewhere (e.g. Munjiza 2004 and Xiang et al. 2009).

In the immersed body approach, two kinds of meshes are used: solid and fluid mesh. On the solid mesh, the constitutive model and Newtonian second law described are solved as outlined in next section. Furthermore, on the fluids mesh, the solid is represented as an immersed body and treated as an additional fluid. Continuity and momentum equations are described in the paper. An adaptive mesh technology is used to refine the mesh around the solid to capture the solid's shape.

Solid phase: 


\section{Governing equations}

The motions of elements are influenced by the forces acting on elemental nodes, internal nodal force, $\mathbf{f}_{\text {int }}$, external nodal force, $\mathbf{f}_{\text {ext }}$, and drag forces $\mathbf{f}_{\text {drag }}$. The constitutive equation influences the deformation of the material through the Cauchy stress tensor in the linear momentum equation given by,

$$
M \frac{\partial \mathbf{u}_{\mathbf{s}}}{\partial t}+\mathbf{f}_{\mathrm{int}}=\mathbf{f}_{d r a g}+\mathbf{f}_{c}+\mathbf{f}_{e x t}
$$

Internal nodal forces are given by

$$
\mathbf{f}_{\mathrm{int}}=\int_{v^{(n)}} \frac{\partial \mathbf{N}}{\partial \mathbf{x}} \mathbf{T} d v
$$

External nodal forces are given by

$$
\mathbf{f}_{e x t}=\int_{v^{(e)}} \mathbf{N b} d v+\int_{v^{(e)}} \mathbf{N t} d a
$$

Where $\mathbf{b}$ is body force, defined by $\mathbf{b}=\left[\begin{array}{lll}b_{x} & b_{y} & b_{z}\end{array}\right], \mathbf{t}$ is surface traction force.

Nodal drag force is given by

$$
\mathbf{f}_{d r a g}=\int_{V_{s}} \frac{\rho_{f}}{\Delta t} \alpha_{f} \alpha_{s}\left(\mathbf{u}_{\mathbf{f}}-\mathbf{u}_{\mathbf{s}}\right) d V
$$

where $\alpha_{f}$ is the volume fraction of fluid in the cell, $\alpha_{s}$ is the volume fraction of solid in the cell, $\alpha_{f}+\alpha_{s}=1$ and $\rho_{f}$ is the fluid density, Fluid velocity vectors is defined by $\mathbf{u}_{f}, \Delta t$ is time step of fluid. It should be noted here $\mathbf{f}_{\text {drag }}$ is non-zero at interface between solid and fluid $\alpha_{s}$ is 1 on solid meshes.

Nodal mass matrix is given by,

$$
\mathbf{M}=\int_{V_{0}} \rho_{0} \mathbf{N N}^{T} d V
$$

Since mass is conserved, the mass is calculated based on the initial configuration and $\rho$ is the density of material. The external nodal forces can be formed of normal contact forces and sliding frictional forces.

\section{Contact forces}

In FEMDEM, a penalty function method is employed to calculate the normal contact force when the two particles are in contact. The penalty function method in its classical form assumes that two particles penetrate each other. The elemental contact force is directly related to the overlapping area of the finite element in contact. The distributed contact force approach takes into account the shape and the size of the overlap area in order to be distributed among the surrounding nodes. Munjiza, (2004) showed that integration over finite elements was equivalent to integration over finite element boundaries and that the contact force is given by

$$
\boldsymbol{f}_{c}=\sum_{i=1}^{n} \sum_{j=1}^{m} \int_{\Gamma_{\beta_{c_{i}} \cap \beta_{t_{j}}}} \boldsymbol{n}_{\Gamma_{\beta_{c_{i}} \cap \beta_{t_{j}}}}\left(\varphi_{c_{i}}-\varphi_{t_{j}}\right) d \Gamma
$$

where $\beta_{\mathrm{c}}$ and $\beta_{\mathrm{t}}$ are the contactor and target discrete elements, respectively, $\mathbf{n}$ is the outward unit normal to the boundary of the overlapping area. The integration over finite element boundaries can be 
written as summation of integration over the edges of finite elements as

$$
\boldsymbol{f}_{c, e d g e}=\frac{1}{\boldsymbol{u}^{2}} \boldsymbol{u} \int_{0}^{L} \boldsymbol{p} \varphi(v) d v
$$

where $\boldsymbol{p}$ is the penalty term. The term $\mathbf{u}^{2}$ comes from the fact that vector $u$ is not unit vector. The calculated elemental contact force is then distributed around the nodes surrounding the contact in order to preserve the system from artificial stress concentration.

\section{Cauchy stress}

The Neo-Hookean material model is an extension of the isotropic linear law (Hooke's law) to large deformations. If the material is assumed viscoelastic, the Cauchy stress can be obtained using the equation below

$$
\mathbf{T}=\frac{\mu}{J}(\mathbf{B}-\mathbf{I})+\frac{\lambda}{2 J} \operatorname{trace}(\mathbf{B}-\mathbf{I}) \mathbf{I}+2 \eta \mathbf{D}
$$

where $\mathbf{B}$ is the left Cauchy-Green tensor $\mathbf{B}=\mathbf{F} \mathbf{F}^{\mathbf{T}}, \mathbf{F}$ is the deformation gradient tensor, $\mu$ and $\lambda$ are two Lamé constants related to Young's modulus and Poisson's ratio.

\section{Fluid phase}

In this work, we used an open source CFD code, Fluidity, as fluid solver to couple with the FEMDEM code Y3D, Fluidity ${ }^{2}$ is a three dimensional Finite Element code for solving the Navier Stokes equations. It is a powerful generic multi-phase CFD code that has been further developed by the authors to handle fluid-structure interaction. The combination of Fluidity together with the Y3D forms the basis of our multi-physics modelling capability. Fluidity employs a wide variety of solving and optimization techniques. Among the most important optimization for our approach was the adaptive tetrahedral mesh optimization that was developed and implemented, as seen in (Pain et al., 2001). The capability of concentrating resolution where it is needed is a key element in describing the solid-fluid interface, and reducing the computational cost.

\section{Coupling of Fluidity with Y3D}

The implementation of the solid's presence within a domain of fluids employs a multi-fluid approach. Each solid particle is modelled as another material with set geometric characteristics, position, and velocity. With appropriate parameterization, drag source terms in the momentum equations provide the approximate description of the boundary layer surrounding the solid, and thus a solution is found. The volumetric drag terms obtained can be integrated to obtain the total force exerted by the fluid onto the solid particle. These fluid forces can then be calculated in the solids solver (Eq 4).

The aim is to solve the particle position and velocity with an external Lagrangian particle solver FEMDEM (Munjiza, 2004) that also receives the fluid-imposed forces. This can provide stress analysis within the fluid-bound particles and structures and has the potential capability to allow particles to fracture and fragment. All of these particle motion characteristics should be straightforwardly captured by the multi-material approach. (Pain et al., 2001).

\section{Momentum equations}

There are three materials to be dealt with. They are the air, the water, and the solid. For simplicity, the two fluid materials will be considered together as one.

The momentum equation for the fluid material can be expressed by,

$$
\alpha_{f} \rho_{f}\left[\frac{\partial \mathbf{u}_{f}}{\partial t}+\mathbf{u}_{f} \nabla \cdot \mathbf{u}_{f}\right]=\nabla p+\nabla \cdot \overline{\bar{\tau}}+\mathbf{F}_{d r a g}-\rho_{f} \mathbf{g}
$$

The viscous tensor is given by $\bar{\tau}, p$ is the pressure, $\mathbf{F}_{d r a g}$ represents the drag force per unit volume acting on the fluid, $\mathbf{F}_{d r a g}=\frac{\alpha_{f} \alpha_{s} \rho_{f}}{\Delta t}\left(\mathbf{u}_{s}-\mathbf{u}_{f}\right)$, the total drag force $\mathbf{F}_{t, d r a g}$ on fluid cell is the

\footnotetext{
${ }^{2}$ For more information, please see AMCG website -- http://amcg.ese.ic.ac.uk
} 
volume integral of $\mathbf{F}_{d r a g}, \mathbf{F}_{t, d r a g}=\int_{V_{f}} \frac{\alpha_{f} \alpha_{s} \rho_{f}}{\Delta t}\left(\mathbf{u}_{s}-\mathbf{u}_{f}\right) d V . \mathbf{F}_{t, d r a g}$ is equal and opposite to the drag force on solid, $\mathbf{f}_{\text {drag }}$.

The continuity equation is

$$
\nabla \cdot \mathbf{u}_{f}=\frac{\partial \alpha_{s}}{\partial t}
$$

\section{Interface tracking}

A volume conservative interface tracking scheme has been developed by Dr Mindel (Mindel, et al 2007). Tracer fields are used to define the Multi-Fluid approach are solved using Control Volume Finite Elements. Using Hyper-C compressive advection scheme, interface sharpness is ensured.

$$
\frac{\partial S}{\partial t}+\mathbf{u}_{f} \cdot \nabla S=0
$$

Where $\mathrm{S}$ is the volume fraction of water indicator, $S \in[0,1]$

\section{RESULTS}

Numerical simulation of a sphere falling through a fluid

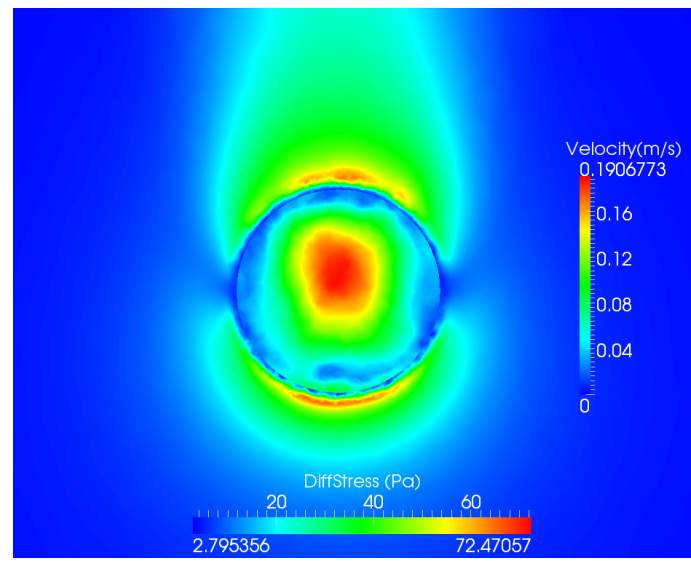

(a)

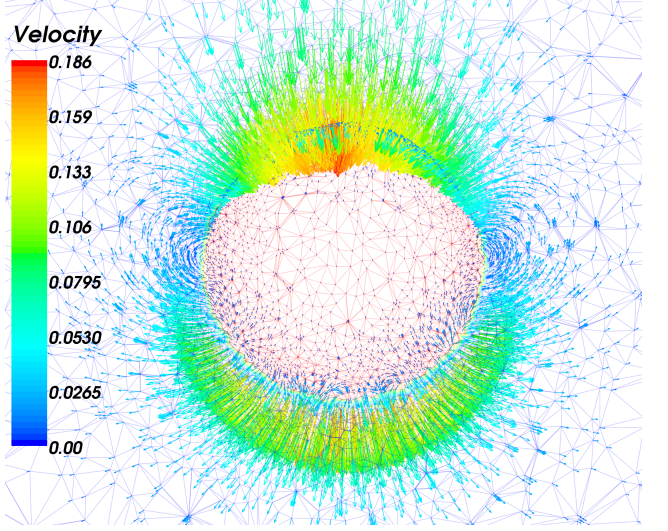

(c)

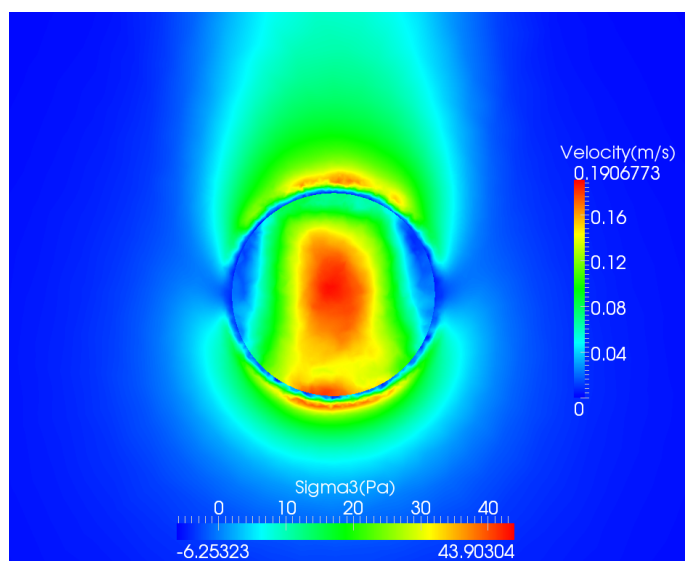

(b)

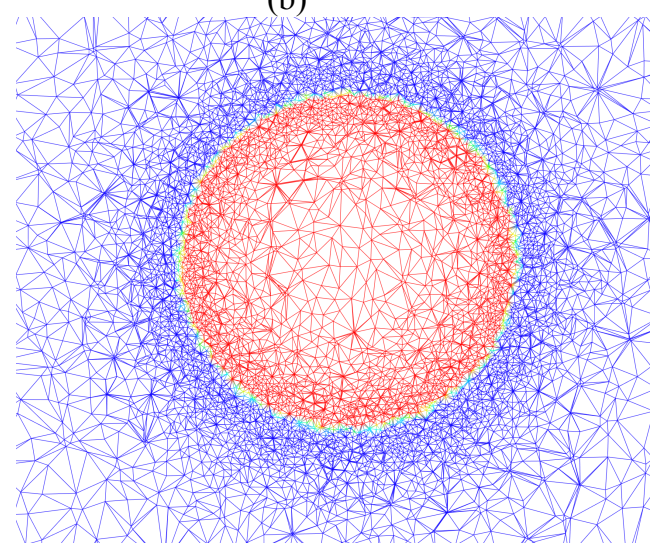

(d)

Figure 1. Two-way coupling Benchmark test of terminal velocity: a sphere falling through water under gravity. Figures show cut planes through mid-plane of domain when the sphere is reaching terminal velocity. (a) and (b): two scalar fields are shown in this figure: scalar of velocity magnitude shows fluid velocity, scalars of Sigma 3 and differential stress show stress response during falling; (c): fluid velocity field is shown; (d) solid volume fraction cut plane shown as mesh, solid volume fraction varies from 0 (blue) to 1 (red), it also shows mesh refinement around the sphere. The terminal velocity agrees with experimental data and the error is within $0.5 \%$. 
A sphere with diameter $0.2 \mathrm{~m}$, density $2,000 \mathrm{~kg} / \mathrm{m}^{3}$ is initially located in a computational domain with zero initial velocity. The fluid with density $1950 \mathrm{~kg} / \mathrm{m}^{3}$, viscosity $1.0 \times 10^{-2}$ is initially set at rest. A no-slip boundary condition is used. The sphere accelerates and soon after reaches a terminal velocity of 0.19 before it impacts with the bottom of the vessel. Numerical results (terminal velocity) are in good agreement with empirical results presented by (Clift, 1978). Figure 1d also shows fluid adaptive mesh around the sphere, finer mesh near surface. It is worth mentioning that as Y3D was used as solid solver, the deformation within the sphere can be simulated. Figure $1 \mathrm{a}$ and $1 \mathrm{~b}$ show least principal stress $\left(\sigma_{3}\right)$ indicating tensile stresses, and differential stress $\left(\sigma_{1}-\sigma_{3}\right)$ when the sphere reaches terminal velocity. When the sphere is dropping through the fluid, it pushes the fluid and then generates higher pressure in front of it. The complementary reverse effect also occurs as the fluid compresses/flattens the sphere, therefore generating differential stresses which are maximum in the middle of the sphere. Figure 1c also shows that fluid flow passes around the sphere and generates shear forces acting on the leading or frontal surface of the sphere. This causes tensile stress to act at the middle of the frontal surface of the sphere. The results are in qualitative agreement with the analytical solution.

\section{Numerical test of 5-CORE-LOC ${ }^{\mathrm{TM}}$ Units sliding into water}

To simulate wave breaking on armour units, a simulation test of regular wave breaking on two armour units resembling the CORE-LOC ${ }^{\mathrm{TM}}$ shape that were fixed to a slope was carried out. Preliminary results of this one-way coupling of waves breaking over unit geometries were shown in a coastal structures conference paper (Xiang 2007). The armour units are at rest and no force is exchanged between fluids and solids.

This paper presented a two-way coupling of wave breaking on armour units. A single wave is generated by water column collapsing and five CORE-LOC ${ }^{\mathrm{TM}}$ shape armour units are loosely packed and are free to slide into water with no friction being applied for this simple case. The wave quickly approaches the armour units, then breaks on them. Figure 5 also shows the fluids flow around the armour units and stress waves propagate in the armour units when they collide with each other and with the slope. The results appear quite realistic and are in qualitative agreement with the expected flows. In future, two-way fully coupled Y3D with Fluidity has considerable applications to industrial problems.

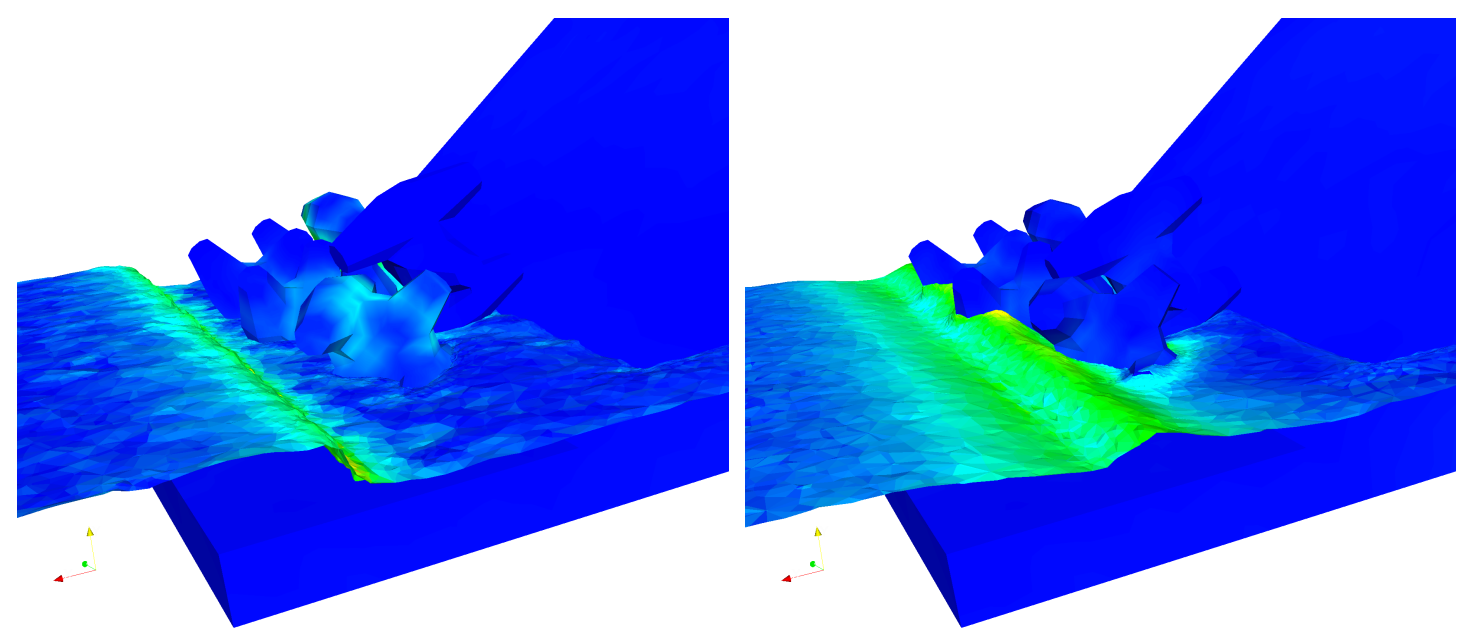

Figure 2. Two-way coupled motion of 5 CORE-LOC ${ }^{\mathrm{TM}}$ concrete units falling through water, with gravitational acceleration, modelled with Y3D/Fluidity. 

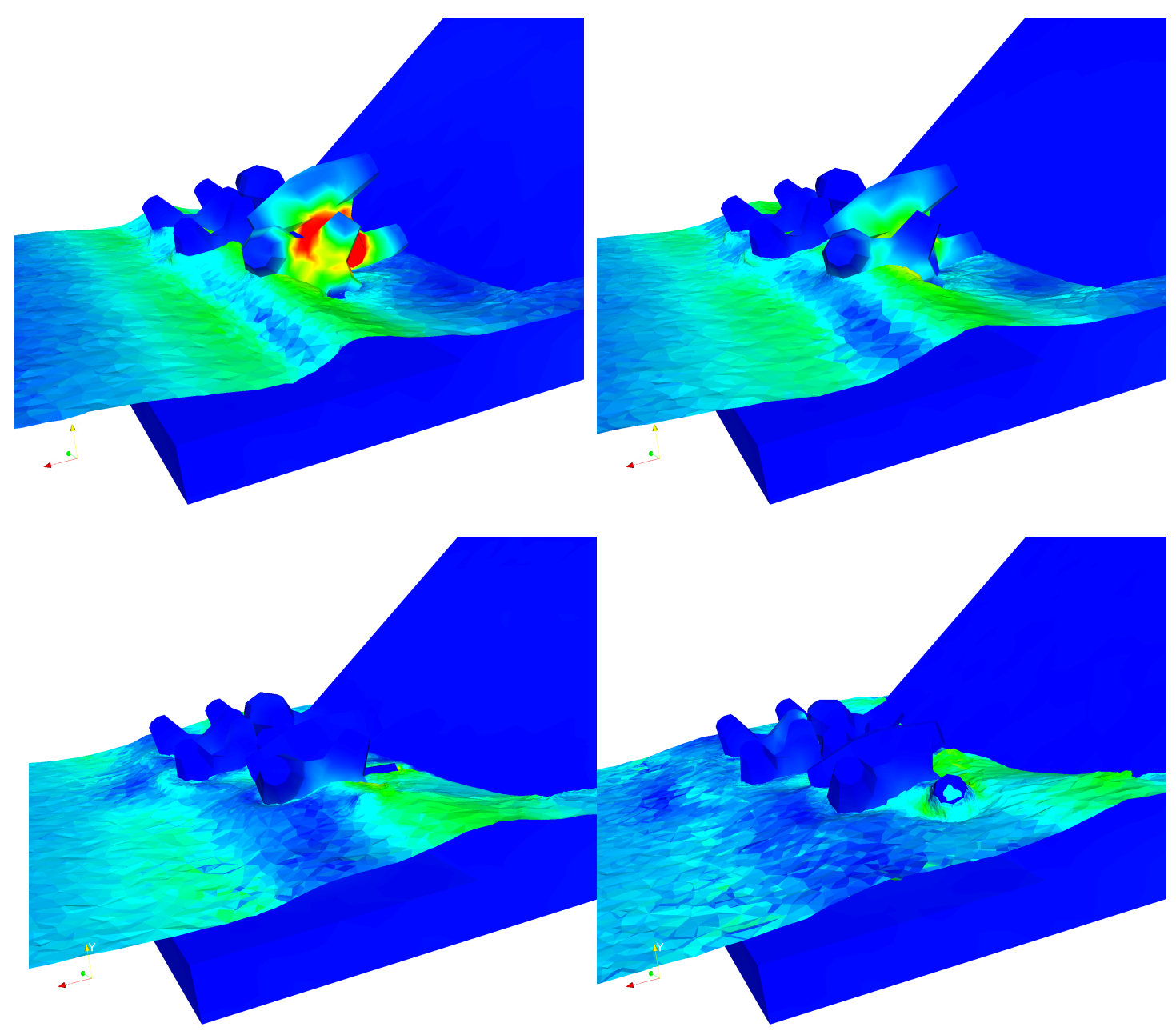

Figure 2 (continued). Two-way coupled motion of 5 CORE-LOC ${ }^{\mathrm{TM}}$ concrete units falling through water, with gravitational acceleration, modelled with Y3D/Fluidity.

\section{SIMULATION TOOLS}

\section{POSITIT/Y3D-R - a new tool for depositing rocks and units}

POSITIT is new tool for introducing particles into a computational domain. In this paper we briefly outline how it has been used in the context of armour unit placements and creation of realistic armour layers. The code is designed to be a versatile i.e. generic depositing tool, which can be used widely for industrial applications such as in particle technology. The program is designed to be compatible with a FEMDEM solver.

As mentioned in previous section, FEMDEM method is capable of simulating not only complex shape but also deformable body. However, the main drawback is high CPU cost which hinder FEMDEM from simulating a full breakwater system formed of over thousands units. Based on full FEMDEM code Y3D_D, rigid version of FEMDEM code, Y3D_R was developed recently. The main difference between $Y \overline{3}$ D_D and Y3D_R is Y3D_R only simulates rigid body and solid deformation is neglected. The time step of Y3D_R is related to whole particle/unit body. On the other hand, the time step of Y3D_R is related to smallest element in particle/unit meshes. Therefore, Y3D_R is much faster than Y3D_D, e.g. if a CORE-LOC ${ }^{\mathrm{TM}}$ unit is formed of $\sim 2500$ elements, Y3D_R is about 50 times faster than Y3D_D. Coupled POSITIT/Y3D_R is capable of simulating a realistic armour unit layer.

POSITIT/Y3D-R allows the user to choose any shape of particle, e.g. any rock or armour unit shape, and to position their centres with user-defined grid file in a predefined container geometry (e.g. rough underlayer with walls, see Figure 3). The particles begin to pile up mechanically as they are caught in the container. At the end of the run, if the particles have come to rest, the particles are touching and in static force equilibrium. A post-process analysis code was developed to analyse 
existing packs in terms of unit max contact force, contact number and stereographic plot. We apply POSITIT/Y3D-R to generate an armour unit layer of 242 CORE-LOC $^{\mathrm{TM}}$ units (21 rows, 11 of which have 12 units and 10 of which have 11 units) on a rough underlayer with 3436 rocks (see Figure 3 ). We also analyse maximum unit contact force by choosing maximum contact force between unit and unit or unit and rock (see Figure 3a). Figure 3b also shows contact number has a wide variation contacts with neighbouring units or rock ranging from 2 to 10 in the pack. In Figure 3c, we use a stereographic plot to show the orientation distribution of the nose axes of the placed CORE-LOC ${ }^{\mathrm{TM}}$ unit.

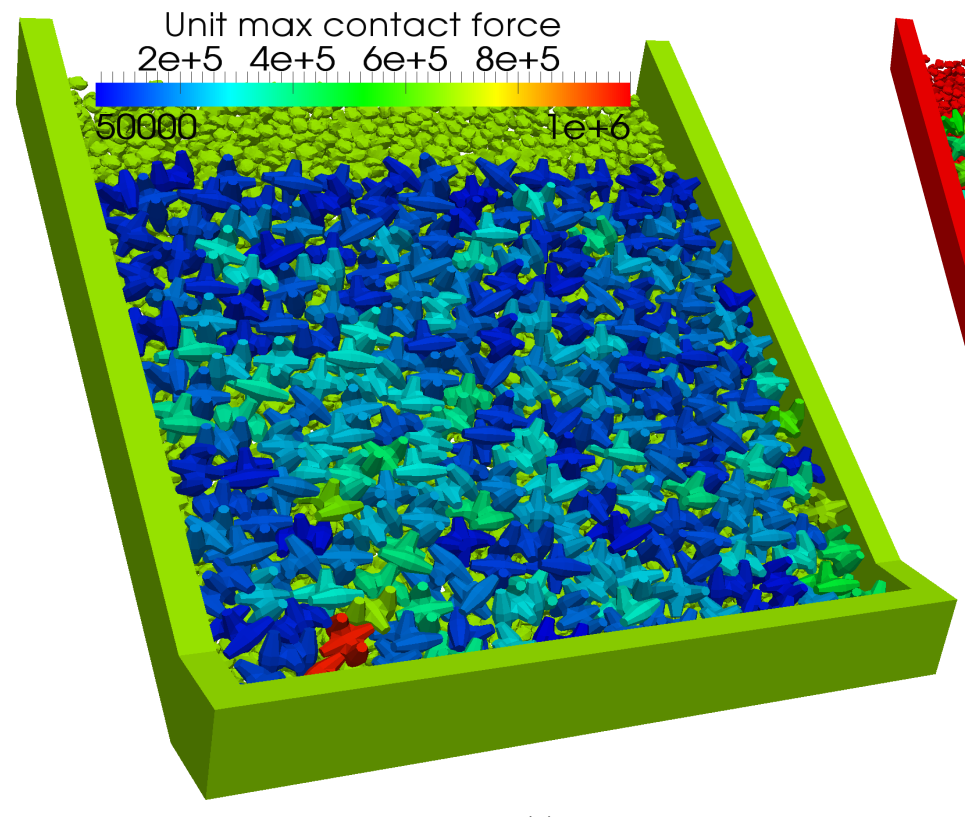

(a) (b)

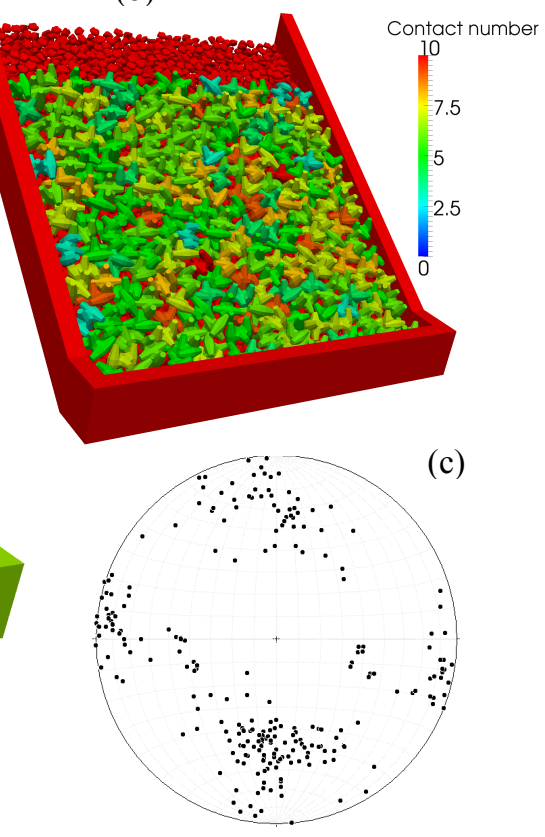

Figure 3a left: a snapshot of an armour layer of 242 CORE-LOC ${ }^{\mathrm{TM}}$ units, color represents unit maximum contact forces; Figure $3 \mathrm{~b}$ shows contact number of each unit; Figure $3 \mathrm{c}$ shows the stereographic plot

\section{CONCLUSIONS}

The paper presents a new FSI approach - the immersed body method which is a full two-way coupling of 3D FEMDEM with Fluidity. This method combines several key technologies: adaptive mesh, super mesh, FEMDEM, interface tracking, and conservative Galerkin projection. The benchmark test of the terminal velocity of a sphere was simulated. Numerical drag force coefficients were compared with experimental data and good agreement was observed. It demonstrated that this method can simulate the fluid-solid interaction and also deformation of solid caused by fluids.

Wave breaking on five armour units was simulated. This showed the capability of modelling the fluid flow around assemblies of particles modelled by FEMDEM. Parallelisation of full two-way coupling of 3D FEMDEM with Fluidity is to be implemented in future work together with large-scale systems.

A more powerful and fully automated placement protocol, POSITIT, was developed to generated realistic large armour unit layers combined with a rigid version of FEMDEM- Y3D-R. A postprocessing analysis code was also developed to analyse existing armour unit layers. These analysis tools include stereographically presented orientation data, coordination number, contact forces between unit-unit or unit-underlayer, as well as other features.

\section{ACKNOWLEDGMENTS}

The authors wish to acknowledge support from EPSRC grant EP/H030123/1, Sogreah (Artelia Group) and W.F. Baird \& Associates.

\section{REFERENCES}

Brown, P.P., Lawler, D.F., 2003, Sphere Drag and Settling Velocity Revisited, Journal of Environmental Engineering, ASCE, 129: 222-231.

Clift, R., Grace, J.R., Weber, M.E., 1978, Bubbles, drops, and particles, Academic press, London. 
Cook, B.K., Noble, D.R., Williams, J.R., 2004, A direct simulation method for particle-fluid systems, Engineering Computations (Swansea, Wales), v21, n2-4, p151-168

Cundall, P.A., Strack, O.D.L. 1979. A discrete numerical model for granular assemblies. Geotechnique, 29:47-65,

Dentale, F., Russo, S. D., Carratelli, E. 2009. Innovative numerical simulation to study the fluid motion within rubble mound breakwaters and the armour stability.

http://www.flow3d.com/pdfs/tp/wat_env_tp/FloSci-Bib01-10.pdf. Published online

Farrell P.E., Maddison J.R., Conservative interpolation between volume meshes by local Galerkin projection, Computer Methods in Applied Mechanics and Engineering, 2011, Vol:200, Pages:89-100

Glowinski, R., Pan, T. W., Hesla, T. I., Joseph, D. D.,Periaux, J., 2001, A fictitious domain approach to the direct numerical simulation of incompressible viscous flow past moving rigid bodies: application to particulate flow, Journal of Computational Physics, v169, n2, p363-426.

Han, K., Feng, Y.T. and Owen, D.R.J., 2007, Coupled Lattice Boltzmann and discrete element modelling of fluid-particle interaction problems, Computers \& Structures, v85, 1080-1088.

$\mathrm{Hu}$, H. H., 1996, Direct simulation of flows of solid-liquid mixtures, International Journal of Multiphase Flow, v22, n 2, p335-352

Mindel, J.E., Collins, G.S., John Paul Latham, J-P, Pain, C.C., Piggott, M.D., and Munjiza, A. 2009.

Towards a numerical wave simulator using the two-fluid interface tracking approach combined with a novel ale scheme. Coastal Structures 2007: pp. 1465-1476.

Latham, J.P., Mindel, J., Guises, R., Garcia, X., Xiang, J., Pain, C., and Munjiza, A., 2007, Coupled FEM-DEM and CFD for coastal structures: application to armour stability and breakage, Fifth International Conference on Coastal Structures, Venice.

Munjiza, A. 2004. The combined finite-discrete element method, John Wiley \& Sons.

Pain, C.C. Umpleby, A.P.; de Oliveria, C.R.E.; Goddard, A.J.H. 2001 Tetrahedral mesh optimisation and adaptivity for steady-state and transient finite element calculations. Computer Methods in Applied Mechanics and Engineering, 190, 3771-96

Pain, C. C., Mansoorzadeh, S. and de Oliveira, C.R.E. and Goddard, A.J.H., 2001a, Numerical modelling of gas-solid fluidised beds using the two-fluid approach, Int. J. Numer. Method. Fluids, 36 91-124

Potapov, A.V.; Hunt, M.L.; Campbell, C.S. 2001, Liquid-solid flows using smoothed particle hydrodynamics and the discrete element method, Powder Technology, v 116, n 2-3, p 204-213

Latham, J-P., Xiang, J and Baird, W.F. 2011. A numerical investigation of the influence of friction and vibration on laboratory scale armour unit layers. Proc. Coastal Structures '11, Yokohama, Japan, to be published.

Xiang, J., Latham, J-P., Zimmer, D., Baird,WF, and Fons M. 2011. Modelling breakwater armour layers and the dynamic response of armour units. Proc. Coastal Structures '11, Yokohama, Japan, to be published.

Xiang, J., Munjiza, A. and Latham, J-P., 2009. Finite strain, finite rotation quadratic tetrahedral element for the combined finite-discrete element method. International Journal for Numerical Methods in Engineering. 79, 946-978

Vire, A., Xiang, J., Latham, J-P, Pain, C. 2012. Modelling of fluid-solid interactions using an adaptivemesh fluid model fluid with a combined finite-discrete element model Ocean Dynamics, under review 